Research Article

\title{
Occipital Nerve Blockade for the Treatment of Occipital Neuralgia-Like Acute Postcraniotomy Headache: A Retrospective Study
}

\author{
Shaoheng Wang $\mathbb{D},{ }^{1,2}$ Xueye Han $\mathbb{D},{ }^{2}$ Chunmei Zhao $\mathbb{D}^{2}, 2$ and Fang Luo $\mathbb{D}^{2}$ \\ ${ }^{1}$ Department of Anesthesiology, Beijing Shijitan Hospital, Capital Medical University, Beijing 100038, China \\ ${ }^{2}$ Department of Pain Management, Beijing Tiantan Hospital, Capital Medical University, Beijing 100070, China
}

Correspondence should be addressed to Fang Luo; 13611326978@163.com

Received 3 February 2021; Accepted 7 April 2021; Published 19 April 2021

Academic Editor: Marcelo M. Valença

Copyright (c) 2021 Shaoheng Wang et al. This is an open access article distributed under the Creative Commons Attribution License, which permits unrestricted use, distribution, and reproduction in any medium, provided the original work is properly cited.

\begin{abstract}
Objective. The therapeutic effectiveness and safety of occipital nerve blockade (ONB) on occipital neuralgia- (ON-) like acute postcraniotomy headache (ON-APCH) was evaluated. Background. Persistent occipital neuralgia is a subclassification of chronic postcraniotomy headache and has been investigated sporadically in previous publications. The long-lasting neuralgic pain significantly impairs postoperative recovery and quality of life. However, little is known regarding ON-APCH and its management. Methods. All data were retrospectively acquired from consultation records and electronic institutional medical documents. Forty-one patients, who developed drug-resistant $\mathrm{ON}-\mathrm{APCH}$ after elective craniotomy and received ONB with lidocaine for diagnoses, were included in this study, all of whom were treated using dexamethasone and lidocaine. Pain intensity and ONB correlated complications and side effects were collected and analyzed at three different time points: before ONB, at 1 day after ONB, and at discharge. Results. Nineteen males and twenty-two females aged $49.6 \pm 15.2$ years were diagnosed with drug-resistant $\mathrm{ON}-\mathrm{APCH}$. The mean NRS was $8.0 \pm 0.9$ before ONB, which later significantly decreased to $2.1 \pm 1.4$ and $1.6 \pm 0.6$ at 1 day after ONB and on discharge, respectively. At 1 month after ONB, thirty patients (73\%) obtained complete pain relief without medication. At 3 months after ONB, only two (5\%) patients had to continue oral medications to maintain pain relief. No adverse effects or complications were observed immediately after, or within 3 months, of the nerve blockade. Conclusions. For drugresistant $\mathrm{ON}-\mathrm{APCH}$, early occipital nerve blockade with dexamethasone and lidocaine is an effective and safe technique, which provides adequate pain relief and may prevent further development of persistent presentation of refractory ON.
\end{abstract}

\section{Introduction}

Occipital neuralgia $(\mathrm{ON})$ is a neuropathic condition that mainly affects the occipital and suboccipital region. The pain is characterized by unilateral or bilateral throbbing, shooting or lancinating episodic aches, presenting along the greater, lesser, and third occipital nerve distribution or, sometimes, in an occipital-temporal-frontal distribution. Hypoesthesia or dysesthesia in the area of occipital nerve innervation, tenderness on palpation, and positive Tinel's sign are some of the major findings of ON. Moreover, severe neuralgic pain often presents with ocular pain, tinnitus, nausea, and dizziness. The accompanying symptoms of $\mathrm{ON}$ occur due to the nervous connections of occipital nerve with vestibulocochlear nerve, glossopharyngeal nerve, vagus nerve, and cervical sympathicus $[1,2]$. Based on the current literature, $8.3 \%$ of all facial pain is reported to be $\mathrm{ON}$, with an incidence of 3.2 per 100,000 people [3]. $90 \%$ of all ON is correlated with pathophysiological changes of the greater occipital nerve, and the other $10 \%$ is due to the damage of lesser occipital nerve. The third occipital nerve rarely results in ONs [4]. Greater occipital nerve and lesser occipital nerve receive sensory fibers from $\mathrm{C} 2$ and $\mathrm{C} 2 / \mathrm{C} 3$ nerve roots, respectively. Typical causes of ON include nerve entrapment, trauma, inflammation, and whiplash [5].

Studies suggest that, following craniotomy, patients are likely to develop persistent $\mathrm{ON}$, which is an uncommon subclassification of chronic postcraniotomy headache 
(PCH). According to the Headache Classification Committee of the International Headache Society (IHS), the International Classification of Headache Disorders, 3rd edition, PCH is defined as acute or chronic headache attributed to craniotomy [6]. Pericranial muscular adherence and aseptic meningitis are believed to be two major factors leading to $\mathrm{PCH}$ [7]. The characterization of $\mathrm{PCH}$ varies in many ways. Symptoms are described as incisional [8], throbbing [9], pressing [10], and sometimes neuropathic [11]. Levo et al. noticed that, after acoustic neuroma removal, some chronic PCHs originate from occipital nerve innervation and present as neuralgic headache in an occipital-temporal-frontal distribution [12]. In 2009, Schankin et al. reported that, 5 out of 30 patients with chronic PCH fit the diagnostic criteria of $\mathrm{ON}$ and presented the first report of persistent ON after acoustic neuroma surgery [13]. Thereafter, Ducic et al. reported that persistent drug-refractory ON following acoustic neuroma resection can be relieved by occipital nerve excision [14]. Ducic et al. described that 6 out of 7 patients experienced pain reduction and improvement of more than $80 \%$ on migraine index and quality of life [14]. This finding confirmed that $\mathrm{ON}$ following acoustic neuroma surgery was associated with a nerve entrapment or neuroma compression due to surgical incision [14]. Additionally, a recent study reported that nerve allograft was positive for drug-resistant persistent $\mathrm{ON}$ caused by lesser occipital nerve damage, during a retrosigmoid craniotomy [15]. Investigations suggest that persistent pain caused by intraoperative impairment of occipital nerves seems to be an uncommon but intractable problem.

Conservative treatments failed in most of the above cases. Most patients must undergo surgical management for persistent ON. It is assumed that early management of ONlike acute postcraniotomy headache (ON-APCH) after craniotomy might better relieve pain and help prevent the development of persistent ON. However, based on the current literature, little is known about $\mathrm{ON}-\mathrm{APCH}$ and its management. In this study, medical data of 41 patients who developed drug-resistant acute $\mathrm{PCH}$, similar in nature to occipital neuralgia, within 7 days after craniotomy, and received occipital nerve blockade (ONB) using dexamethasone and lidocaine, were retrospectively collected and analyzed. The therapeutic effectiveness and safety of ONB on $\mathrm{ON}-\mathrm{APCH}$ was evaluated.

\section{Patients and Methods}

2.1. Population and Eligibility. Patients' medical documents and case records between January 2012 and December 2020 were reviewed. Patients who fulfilled the following criteria were included in this study: underwent elective craniotomy for intracranial disease, asked for a consultation with the institutional pain management center, diagnosed with ONAPCH following craniotomy within 1 week as described by the Headache Classification Committee of the IHS, the International Classification of Headache Disorders (version 2 , 3-beta, and 3 based on the time of diagnosis) $[6,16,17]$, presented with severe pain that could not be relieved by oral analgesics, and received therapeutic ONB by pain physicians. Patients with any of the following criteria were excluded from the study: confirmed with ON before craniotomy, underwent other invasive treatment for ON$\mathrm{APCH}$ before $\mathrm{ONB}$, and had incomplete clinical records.

2.2. Diagnosis and Treatment. For patients who underwent elective craniotomy, routine postoperative analgesia through oral analgesics, and patient-controlled intravenous analgesia (PCIA), containing opiates and antiemetics, were given as recommended, on the first postoperative day [18]. PCIA was removed before the end of the second postoperative day. When patients reported a drug-resistant postoperative pain or headache after craniotomy, after intracranial pathophysiological changes were ruled out, consultation with a pain physician was asked by the neurosurgeons. If symptoms and examinations suggested ON, diagnostic blockade of the greater and/or lesser occipital nerve was performed by the pain physician to confirm ON-APCH. Therapeutic ONB was performed for patients who responded positively to the diagnostic blockade. After nerve blockades, oral analgesics were continued or discontinued as per the evaluation of the pain physician.

2.3. Nerve Blockade Technique. Patients were asked to be seated with their head forward, positioning the ears in front of the body's vertical line. The injection point of greater occipital nerve was identified by palpation, and superior nuchal ridge was palpated to confirm occipital artery pulsation. Occipital nerve is located medial to the occipital artery, approximately $2 \mathrm{~cm}$ lateral and $2 \mathrm{~cm}$ inferior to occipital protuberance. After sterilization, a $5 \mathrm{ml}$ syringe, with 25-27-gauge needle of approximately $1-1.5$-inch, was orthogonally inserted to a depth of $3-4 \mathrm{~mm}$ until stopped by the periosteum. Once the needle was stopped, it was withdrawn $1 \mathrm{~mm}$ backward and then reoriented cephalad. A fanlike injection was performed after aspiration. For lesser occipital nerve blockade, the puncture site was localized approximately $3-4 \mathrm{~cm}$ lateral and $0.5-1 \mathrm{~cm}$ caudal to the former site. The optimal position was confirmed by palpation as well. Sterilization, aspiration, and injection procedure were identical to ONB. For diagnostic blockade, 1\% lidocaine was administered at each puncture point. As to therapeutic blockade, the injection was formulated with $1 \%$ lidocaine and dexamethasone $(4 \mathrm{mg})$, diluted with normal saline. The solution was injected into the affected nerves based on the evaluation of pain distribution and intensity $[19,20]$.

2.4. Data Collection and Evaluation. Data were collected by two research assistants by reviewing consultation records, case records, and institutional electronic medical record system. Preoperative baseline demographics were obtained from the electronic medical record system, which included gender, age, preoperative comorbidities, or headache disorders. Perioperative data were collected from the operation and anesthesia records, including preoperative regional management, side of surgical incision (right or left), reason 
TABLE 1: Barrow Neurological Institute pain inventory.

\begin{tabular}{lc}
\hline BNI degrees & Explanations \\
\hline BNI I & No pain without medications \\
BNI II & Mild pain not requiring medications \\
BNI III & No or controlled pain with medications \\
BNI IV & Improved pain cannot be relieved by medications \\
BNI V & Persistent pain cannot be relieved by medications \\
\hline
\end{tabular}

BNI: Barrow Neurological Institute pain inventory.

TABLE 2: Baseline demographics and perioperative data $(n=41)$.

\begin{tabular}{lc}
\hline Characteristics & Values \\
\hline Age (years) & $49.6 \pm 15.2$ \\
\hline Gender & $22(53.7 \%)$ \\
Female & $19(46.3 \%)$ \\
Male & $4(9.8 \%)$ \\
Preoperative anxiety and/or depression & \\
\hline Preoperative headache & $3(7.3 \%)$ \\
Tension-type headache & $2(4.9 \%)$ \\
Migraine & \\
\hline Preoperative regional management & $17(41.5 \%)$ \\
Scalp nerve blockade & $19(46.3 \%)$ \\
Scalp infiltration & $5(12.2 \%)$ \\
N/A & \\
\hline Side of surgical incision & $21(51.2 \%)$ \\
Left & $20(48.8 \%)$ \\
Right & $18(43.9 \%)$ \\
\hline Etiology for surgery & $13(29.3 \%)$ \\
Tumor resection & $10(22.0 \%)$ \\
Decompression & \\
Others & $23(56.1 \%)$ \\
\hline Surgical approach & $16(39.0 \%)$ \\
Retrosigmoid & $2(4.9 \%)$ \\
Suboccipital & $6.3 \pm 1.1$ \\
Others & \\
Operation duration (hours) & \\
\hline
\end{tabular}

for surgery, surgical approach, and duration of operation. Data on time of onset of ON, symptoms of pain presentation, pain intensity, analgesics requirement (required or not required), time-to-treat (defined as the time interval from the onset of pain to ONB), side effects, or complications attributed to ONB were collected from case records. Routine revision and follow-up were scheduled one day after consultation. During hospitalization, a pain specialist made regular follow-up visits, one day after consultation, and before discharge, in order to observe the effects of treatment. After discharge, routine follow-up through telephone interviews were scheduled at 1 and 3 month(s) after ONB. Pain intensity was determined using a Numerical Rating Scale (NRS) during hospitalization, in which 0 indicated no pain and 10 indicated the worst pain imaginable, and the Barrow Neurological Institute (BNI) pain inventory after discharge, which is shown in Table 1.

2.5. Statistical Analyses. All data were analyzed using SPSS version 25.0. Normality was examined using the Shapiro-Wilk test. Normally distributed continuous variables

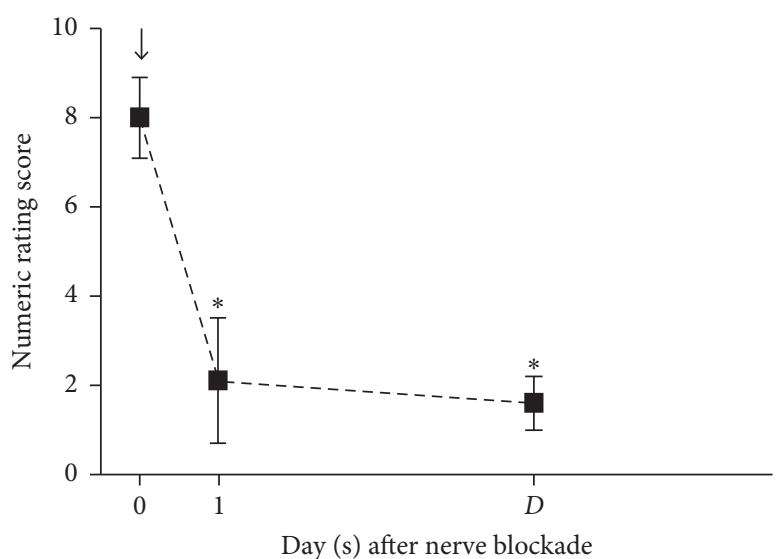

Figure 1: Numeric rating scores in 41 patients before and after occipital nerve blockade. Data at each time point were presented with mean and standard deviation. $\downarrow$ : occipital nerve blockade. $*, P<0.001$ vs. baseline. $\mathrm{D}$ : discharge.

were presented with mean and standard deviation. Skewed data were presented with median (range). Categorical variables were recorded as frequency (percentage). NRS before and after ONB was compared using the one-way repeated-measures analysis of variance (ANOVA). To evaluate the influence of timing of ONB on therapeutic effectiveness, two subgroups were formed based on timeto-treat ( $\leq 7$ or $\geq 8$ days) with two-way repeated-measures ANOVA. $P<0.05$ was determined for statistical significance.

\section{Results}

We retrospectively reviewed institutional medical records from January 2012 to December 2020. A total of 51 patients fulfilled the inclusion criteria; four were confirmed to have pre-existing $\mathrm{ON}$, and one had received acupuncture in the suboccipital region before consultation. Incomplete data due to loss of follow-up was recorded in five patients. Ultimately, 41 patients were included in the present study. Baseline demographics and perioperative data of eligible patients are shown in Table 2.

After craniotomy, ipsilateral ON-APCH was found in 38 of the 41 patients. 3 patients developed bilateral ON-APCH postoperatively. The onset of symptoms was $3.5 \pm 1.3$ days after craniotomy. 22 patients complained of throbbing and/ or stabbing pain. The other 19 patients described the pain to be indistinguishable. The median time-to-treat was $6(2-11)$ days. Unilateral greater and lesser occipital nerve blockade was performed in 13 and 17 patients, respectively. Unilateral blockade for both greater and lesser was performed in 8 patients. 3 patients received bilateral greater occipital nerve blockade.

The difference in mean NRS at each time point was statistically different $(\mathrm{df}=1.4, F=444.3, P<0.001)$. NRS was $8.0 \pm 0.9$ before ONB, $2.1 \pm 1.4$ at one day after ONB, and $1.6 \pm 0.6$ at discharge (Figure 1). There was a statistically significant difference in NRS at 1 day after ONB and at discharge, compared to NRS before ONB. 


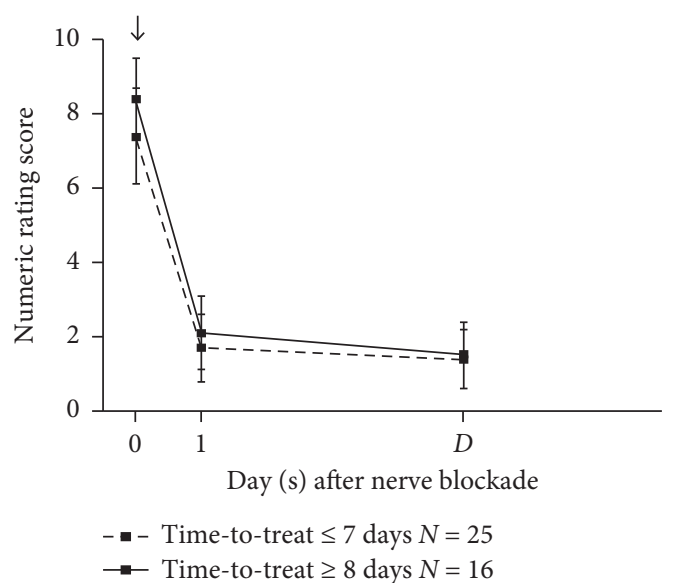

Figure 2: Numeric rating scores for the time-to-treat. Data at each time point were presented with mean and standard deviation. Time-to-treat: time interval from pain onset to occipital nerve blockade. $\downarrow$ : occipital nerve blockade. Interaction concerning timeto-treat was not detected by the two-way repeated-measures ANOVA $(\mathrm{df}=2, F=1.984, P=0.152)$. D: discharge.

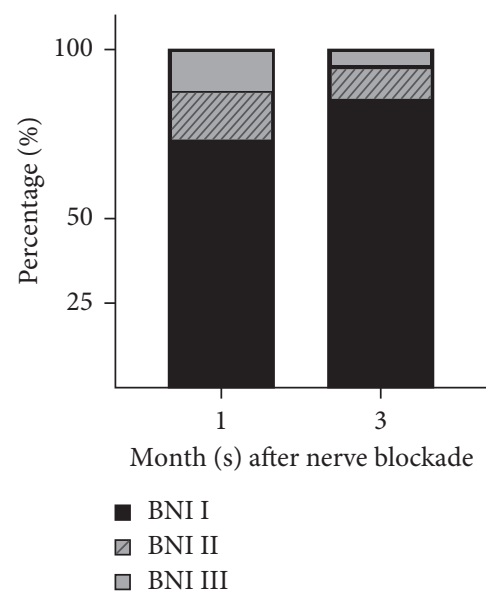

Figure 3: Pain intensity at 1 and 3 month(s) after occipital nerve blockade. BNI: Barrow Neurological Institution pain inventory.

All patients received analgesics administration before ONB. $25(61 \%)$ patients discontinued analgesics one day after ONB, while only 11 (27\%) patients continued analgesics use after discharge.

To determine the impact of ONB timing on therapeutic effectiveness, patients were divided into two groups based on time-to-treat ( $\leq 7$ or $\geq 8$ days). Twenty-five patients received ONB within 7 days after ON onset, while the other sixteen underwent treatment beyond 8 days. NRS before ONB, at 1 day after ONB, and at discharge were compared between the two subgroups. Although longer time-to-treat was associated with a higher NRS at each time point, the difference between the groups were not statistically significant (Figure 2) $(\mathrm{df}=2, F=1.984, P=0.152)$. NRS fell analogously at each time point, regardless of the timing of ONB administration after craniotomy.

The effectiveness of ONB during follow-up period is described in Figure 3. All 41 patients received stable pain relief with or without medication (BNI I-III) during the follow-up period. At 1 month after ONB, thirty (73\%) patients experienced no pain without medication (BNI I), while five (12\%) patients had to take oral analgesics (BNI III). Six (15\%) patients experienced mild pain that did not require medication (BNI II). At 3 months after ONB, only two (5\%) patients required medication to maintain pain relief (BNI III). Thirty-five (85\%) patients experienced no pain without medication (BNI I), while four (10\%) patients experienced mild pain that did not require analgesics (BNI II).

Neither immediate nor extended adverse effects or complications, including transient dizziness, intravenous injection, sudden unconsciousness, cardiovascular reactions, peri-injection alopecia, gastric ulcers, and other steroid-related adverse effects were observed after ONB throughout the study period.

\section{Discussion}

In this study, we report the therapeutic effectiveness and safety of occipital nerve blockade (ONB) with dexamethasone and lidocaine in 41 patients with drug-resistant ON$\mathrm{APCH}$, by reviewing consultation records and electronic medical data between January 2012 and December 2020. ON following craniotomy is deemed an uncommon subclassification of PCH. Ever since Schankin et al. first subclassified ON following craniotomy [7], persistent ON following craniotomy has occasionally been reported. However, little has been reported regarding acute occipital neuralgia-like postcraniotomy headache. To the best of our knowledge, this is the first study on ON-APCH and the therapeutic effectiveness and safety of ONB with dexamethasone and lidocaine in treating $\mathrm{ON}-\mathrm{APCH}$.

In line with the management of $\mathrm{ON}$, treatment of $\mathrm{ON}$ $\mathrm{APCH}$ includes conservative treatment and interventional procedures. Conservative treatments including physical therapies, rest, heat, massage, and nonsteroidal anti-inflammatory drugs (NSAIDs) remain the first-line therapy. For drug-resistant patients, interventional procedures are performed. In the current study, significant improvement was observed in all patients after therapeutic ONB procedure. Compared to the NRS before ONB, mean NRS decreased by $74 \%$ at 1 day after ONB and $93 \%$ at discharge. Approximately $40 \%$ of patients stopped oral analgesic medications at day 1 after ONB. The result is in line with a previous study on ONB treating drug-resistant ON [21]. Evident pain relief was maintained at discharge $(1.6 \pm 0.6$ on NRS), and only $28 \%$ of patients had to continue oral analgesic for satisfactory pain relief. Oral analgesia was needed by $12 \%$ and $5 \%$ of patients at 1 month and 3 months after $\mathrm{ONB}$, respectively. A majority of patients developed no pain or slight pain without medications during the 3-month follow-up period. No recurrent drug-resistant $\mathrm{ON}$ was observed. In a recent case review, symptomatic pain relief that was maintained for more than 3 months after ONB was reported in $74 \%$ of patients with persistent ON [22]. The effective rate was relatively higher in our findings. This is probably because patients' symptoms were managed within 
6 (2-11) days of onset, in our study. Subgroup analyses revealed that promising therapeutic effect was observed in all patients after ONB regardless of time-to-treat. Though higher NRS before ONB was reported among patients with time-to-treat longer than 7 days, statistical difference was not observed between the two subgroups ( $\leq 7$ or $\geq 8$ days) by two-way repeated-measures ANOVA. The effectiveness of ONB was predictably excellent, as all patients received ONB at an early stage of pain onset (within 11 days), though higher NRS was observed in patients with longer time-totreat. Based on this trend, we speculate that ONB would be less effective in persistent ON. For persistent presentations of $\mathrm{ON}$, pharmacological treatment including serotonin reuptake inhibitors, tricyclic antidepressants and anticonvulsants, and even surgical techniques like occipital nerve excision can be used. None of the 41 patients included in this study developed persistent ON throughout the follow-up period. Our results reveal that early diagnosis and management of ON-APCH provides reliable pain relief and may prevent further development of persistent presentation of refractory ON.

Although imaging guidance was not applied, ONB procedures were performed by skilled physicians, and injection sites were confirmed by anatomical location and physical examinations. Dexamethasone, as recommended and used in previous literatures [21,23], was conservatively administered (not exceed $4 \mathrm{mg}$ ), to avoid excessive systemic absorption of corticosteroids. Throughout the course of the study, side effects or complications of dexamethasone and nerve blockade were not observed, suggesting that ONB is a safe technique for $\mathrm{ON}-\mathrm{APCH}$.

Although the etiology of ON-APCH remains unclear, recent reports suggest perioperative trauma to be the cause of ON [22]. The underlying trauma to the occipital region in retrosigmoidal or suboccipital craniotomy is believed to result in ON. Chronic entrapment of occipital nerves caused by peri-incisional neuroma formation led to nerve injury, which leads to persistent $\mathrm{ON}$ after craniotomy. In this study, all patients with $\mathrm{ON}-\mathrm{APCH}$ responded positively to ONB with lidocaine and dexamethasone. When administered along with lidocaine for nerve blockade, the pharmacological mechanism of dexamethasone mainly involves elimination of local inflammation. This suggests that inflammation of surrounding tissue caused by incisional damage might be an underlying cause of ON-APCH. However, this cannot explain why 3 patients developed bilateral ON after unilateral craniotomy. Occipital nerve excitation due to prolonged occipital muscle traction as well as a fixed position during craniotomy was suspected to promote bilateral ON.

This study has some potential limitations. According to the IHS International Classification of Headache Disorders (3rd version) [17], headaches that are reported to have developed within 7 days after craniotomy should be diagnosed as acute postcraniotomy headache (APCH). Although, APCH mostly occurs at the incision site, or as tension type headache or migraine [17], postcraniotomy patients who developed neuralgic pain in the occipital or suboccipital area were included in this study. Based on clinical presentation and physical examination, we speculate that the patients developed ON after craniotomy. In addition, the outcomes of ONB support the diagnosis of ON. However, it is difficult to diagnose ON based on the current version of classification of headache disorders [17]; therefore, the postcraniotomy pain observed in the present study is classified as ON-APCH. The sample size of our study was limited due to the low prevalence of ON-APCH. Because of its retrospective nature, all patients included in this study were screened for drug-resistant $\mathrm{ON}-\mathrm{APCH}$. Thus, the sample size was further reduced. Therefore, larger, multicentric, prospective research is necessary to identify the clinical presentations, managements, and prophylaxis of this special type of APCH. Moreover, long-term effectiveness of ONB was not investigated due to the lack of a prospective design. Notably, the results of our study should be taken with caution as no control group was set.

\section{Conclusion}

For drug-resistant ON-APCH cases, early ONB with dexamethasone and lidocaine is an effective and safe choice, which provides reliable pain relief and prevents further development of persistent presentation of refractory ON.
Abbreviations
$\mathrm{ON}$ : Occipital neuralgia
TON: Third occipital nerve
PCH: Postcraniotomy headache
IHS: International Headache Society
ONB: Occipital nerve blockade
IRB: Institutional review board
PCIA: Patient-controlled intravenous analgesia
NRS: $\quad$ Numerical Rating Scale
BNI: Barrow Neurological Institute pain inventory
ANOVA: Analysis of variance.

\section{Data Availability}

The datasets used and/or analyzed during the current study are available from the corresponding author on reasonable request.

\section{Ethical Approval}

Ethical approval was obtained from the Institutional Review Board (IRB) of Beijing Tiantan Hospital, Capital Medical University (KY 2019-081-01-1), before the collection of research data.

\section{Consent}

The study is exempt from the requirement of informed consent.

\section{Conflicts of Interest}

The authors declare that they have no conflicts of interest. 


\section{Authors' Contributions}

SHW and XYH contributed equally to this work and should be considered co-first authors. SHW and XYH drafted the manuscript, enrolled participants, and collected data. CMZ participated in statistical analysis of data. FL created and designed the trial. All authors read and approved the final manuscript.

\section{References}

[1] W. F. Kuhn, S. C. Kuhn, and H. Gilberstadt, "Occipital neuralgias: clinical recognition of a complicated headache. A case series and literature review," Journal of Orofacial Pain, vol. 11, no. 2, pp. 158-165, 1997.

[2] P. Vanelderen, A. Lataster, R. Levy, N. Mekhail, M. van Kleef, and J. Van Zundert, "8. Occipital neuralgia," Pain Practice, vol. 10, no. 2, pp. 137-144, 2010.

[3] J. S. H. A. Koopman, J. P. Dieleman, F. J. Huygen, M. De Mos, C. G. M. Martin, and M. C. J. M. Sturkenboom, "Incidence of facial pain in the general population," Pain, vol. 147, no. 1, pp. 122-127, 2009.

[4] S. R. Hammond and G. Danta, "Occipital neuralgia," Clinical and Experimental Neurology, vol. 15, pp. 258-270, 1978.

[5] K. kulkarni, "Occipital neuralgia: a review," Journal of Pain Management \& Medicine, vol. 4, no. 1, 2018.

[6] "Headache classification committee of the international headache society (IHS) The international classification of headache disorders," Cephalalgia, vol. 38, pp. 1-211, 2018.

[7] P. A. S. Rocha-Filho, "Post-craniotomy headache: a clinical view with a focus on the persistent form," Headache, vol. 55, pp. 733-738, 2015.

[8] T. Rimaaja, M. Haanpää, G. Blomstedt, and M. Färkkilä, "Headaches after acoustic neuroma surgery," Cephalalgia, vol. 27, no. 10, pp. 1128-1135, 2007.

[9] J. R. Gee, Y. Ishaq, and N. Vijayan, "Postcraniotomy headache," Headache: The Journal of Head and Face Pain, vol. 43, no. 3, pp. 276-278, 2003.

[10] B. Schaller and A. Baumann, "Headache after removal of vestibular schwannoma via the retrosigmoid approach: a long-term follow-up-study," Otolaryngology-Head and Neck Surgery, vol. 128, no. 3, pp. 387-395, 2003.

[11] H. Batoz, O. Verdonck, C. Pellerin, G. Roux, and P. Maurette, "The analgesic properties of scalp infiltrations with ropivacaine after intracranial tumoral resection," Anesthesia \& Analgesia, vol. 109, no. 1, pp. 240-244, 2009.

[12] H. Levo, G. Blomstedt, T. Hirvonen, and I. Pyykko, "Causes of persistent postoperative headache after surgery for vestibular schwannoma," Clinical Otolaryngology and Allied Sciences, vol. 26, no. 5, pp. 401-406, 2001.

[13] C. Schankin, C. Gall, and A. Straube, "Headache syndromes after acoustic neuroma surgery and their implications for quality of life," Cephalalgia, vol. 29, pp. 760-771, 2008.

[14] I. Ducic, J. M. Felder III, and M. Endara, "Postoperative headache following acoustic neuroma resection: occipital nerve injuries are associated with a treatable occipital neuralgia," Headache: The Journal of Head and Face Pain, vol. 52, no. 7, pp. 1136-1145, 2012.

[15] J. P. Andrews, M. McDermott, and L. Jacques, "Lesser occipital nerve damage after retrosigmoid craniotomy repaired with processed nerve allograft," Interdisciplinary Neurosurgery, vol. 19, Article ID 100592, 2019.

[16] Headache Classification Committee of the International Headache Society (IHS), "The international classification of headache disorders, 3rd edition (beta version)," Cephalalgia, vol. 33, pp. 629-808, 2013.

[17] J. Olesen, "The international classification of headache disorders. 2nd edition (ICHD-II)," Revue Neurologique, vol. 161, no. 6-7, pp. 689-691, 2005.

[18] L. Molnár, É. Simon, R. Nemes, B. Fülesdi, and C. Molnár, "Postcraniotomy headache," Journal of Anesthesia, vol. 28, no. 1, pp. 102-111, 2014.

[19] H. Benzon, C. L. Wu, J. Rathmell et al., Practical Management of Pain, Elsevier, Amsterdam, Netherlands, 5th edition, 2014.

[20] A. Blumenfeld, A. Ashkenazi, U. Napchan et al., "Expert consensus recommendations for the performance of peripheral nerve blocks for headaches-a narrative review," Headache: The Journal of Head and Face Pain, vol. 53, no. 3, pp. 437-446, 2013.

[21] R. Juškys and G. Šustickas, "Effectiveness of treatment of occipital neuralgia using the nerve block technique: a prospective analysis of 44 patients," Acta Medica Lituanica, vol. 25, pp. 53-60, 2018.

[22] E. Martínez-Pías, J. Trigo-López, D. García-Azorín, A. McGreal, and Á. L. G. Peral, "Clinical characteristics and therapeutic results in a series of 68 patients with occipital neuralgia," Pain Medicine, vol. 22, no. 2, pp. 396-401, 2019.

[23] H. T. Benzon, Practical Management of Painp. 52, 5th edition, Elsevier, Amsterdam, Netherlands, 2014. 